\title{
Kein langfristiger Schutz durchs Stillen
}

\author{
Muttermilch in den ersten Lebensmonaten ist fester Bestandteil der \\ aktuellen Empfehlungen zur Allergie- und Asthmaprävention. In einer \\ australischen Studie wurde nun nach dem Langzeiteffekt - nämlich \\ nach rund 40 Jahren - geforscht.
}

n den meisten Allergie- und Asthma-

Geburtskohortenstudien werden die Kinder bis zum Jugendalter, selten bis ins frühe Erwachsenenalter, nachbeobachtet. In der 1968 aufgelegten Tasmanian Asthma Study wurde nun erstmals prospektiv der Effekt einer ausschließlichen Gabe von Muttermilch in den ersten drei Lebensmonaten auf die Entwicklung von Allergie und Asthma über fast 40 Jahre hinweg untersucht.

Die Auswertung der Langzeitdaten von 8.280 Personen ergab, dass ausschließlich gestillte Kinder von atopischen Müttern bis zu einem Alter von sieben Jahren ein leicht vermindertes Asthmarisiko gegenüber Kindern hat- ten, die nicht ausschließlich mit Muttermilch ernährt wurden (Odds Ratio [OR] 0,8). In der Folgezeit kehrte sich

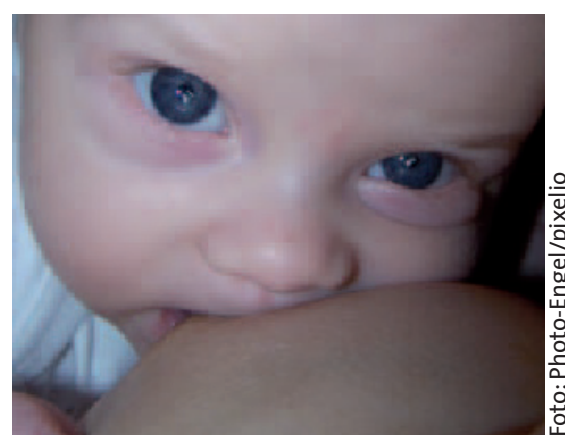

Muttermilch schützt vor Allergie - jedoch nur in den ersten Lebensjahren.

\section{Wheezing durch Selenmangel?}

\section{Als ein möglicher Risikofaktor für die Asthmaentwicklung wird eine zu geringe Selenaufnahme diskutiert. In einer Kohortenstudie wurde der Zusammenhang zwischen Selen-Plasmakonzentration bei Schwangeren und Wheezing der Kinder untersucht.}

D as Spurenelement Selen verfügt als Bestandteil des Enzyms Glutathionperoxidase über starke antioxidative Eigenschaften, eine Verringerung der antioxidativen Kapazität wird als eine der Ursachen für die Zunahme der Asthmainzidenz angesehen. Basierend auf der weiteren Annahme, dass die exogen bedingten Grundlagen für die Entwicklung eines Asthmas häufig in der frühen Kindheit gelegt werden, wurde in einer Geburtskohorte aus Großbritannien der Zusammenhang zwischen Selenstatus und Asthmaentwicklung untersucht.

Es wurden die Selenplasmakonzentrationen sowie die Konzentrationen der Glutathionperoxidase in den Erythrozyten bei 2.000 Schwangeren und ihren 1.924 als Einlinge geborenen Kinder er- mittelt und die Kinder bis zum Alter von fünf Jahren nachbeobachtet. Diese Werte wurden in Beziehung gesetzt zu den Ergebnissen eines Fragebogens zu respiratorischen Symptomen bei 1.282 Kindern im Alter von zwei Jahren und bei 1.167 Kindern im Alter von fünf Jahren. Von den fünfjährigen Kindern konnten 700 zusätzlich im Hautpricktest und 478 spirometrisch zur Feststellung des FEV1 untersucht werden.

Es ergab sich ein inverser Zusammenhang zwischen den Selenwerten der Mütter in der Frühschwangerschaft sowie den Selenwerten des Nabelschnurbluts und Wheezing beim Kind im zweiten Lebensjahr. Im Alter von fünf Jahren waren diese Zusammenhänge wieder verschwunden. Die Glutathionperoxida- das Risikoverhältnis jedoch um: Ausschließlich brustgefütterte Kinder hatten im Alter von 14 Jahren bis zur letzten Nachbeobachtung im Alter von 44 Jahren ein durchgehend erhöhtes Risiko für Asthma (14 Jahre: OR 1,46; 32 Jahre: OR 1,84; 44 Jahre: OR 1,57).

Das Risiko für eine Nahrungsmittelallergie war bei den ausschließlich gestillten Kindern im Alter von sieben Jahren ebenfalls vermindert, im Alter von 44 Jahren stieg bei ihnen aber das Risiko sowohl für eine Nahrungsmittelallergie als auch eine allergische Rhinitis leicht an.

Fazit: Allergiegefährdete Kinder sind durch ausschließliche Brustfütterung in den ersten Lebensmonaten vor frühem Asthma und vor Allergie geschützt. Ein langfristiger Schutz besteht aber nicht.bk

Matheson MC et al. Breast-feeding and atopic disease: a cohort study from childhood to middle age. J Allergy Clin Immunol 2007; 120: 1051-7

sewerte hatten zu keinem Zeitpunkt der Untersuchung einen Einfluss auf respiratorische Symptome bei den Kindern. Auch war insgesamt kein Zusammenhang zwischen Selenwerten und Asthma bzw. einer atopischen Sensibilisierung zu erkennen.

Die Autoren vermuten, dass Selen auch über immunstimulierende Eigenschaften verfügt, die sich insbesondere bei der Abwehr von viralen Infekten bemerkbar machen. Dadurch könnten Kinder mit Selenmangel in der frühen Lebensphase anfälliger für virale Infektionen und damit auch für Wheezing werden.

Fazit: Der Selenstatus der werdenden Mutter sowie des Neugeborenen ist mit Wheezing beim Kind bis zum zweiten Lebensjahr assoziiert. Im Alter von fünf Jahren verschwinden diese Zusammenhänge jedoch wieder.

$b k$

Devereux G et al. Early childhood wheezing symptoms in relation to plasma selenium in pregnant mothers and neonates. Clin Exp Allergy 2007; 37: 1000-8 